ORIGINAL RESEARCH

\title{
Rhabdomyolysis and Hyponatremia: A Cluster of Five Cases at the 161-km 2009 Western States Endurance Run
}

\author{
Jessica Rose Bruso, DO; Martin D. Hoffman, MD; Ian R. Rogers, MBBS; Linda Lee, MS, FNP; \\ Gary Towle, MD; Tamara Hew-Butler, DPM, PhD
}

From the Department of Physical Medicine \& Rehabilitation, University of California Davis Medical Center, Sacramento, CA (Dr. Bruso); Department of Physical Medicine \& Rehabilitation, Department of Veterans Affairs, Northern California Health Care System, and University of California Davis Medical Center, Sacramento, CA (Dr. Hoffman); Sir Charles Gairdner Hospital and University of Western Australia, Perth, WA, Australia (Dr. Rogers); Independent practitioner, Leadville, CO, USA (Ms. Lee); Yosemite Medical Clinic, Yosemite, CA, USA (Dr. Towle); and Exercise Science, Oakland University, Rochester, MI, USA (Dr. Hew-Butler).

\begin{abstract}
Objective.-Five of 400 starters of the 2009 Western States Endurance Run (WSER) were hospitalized with hyponatremia and rhabdomyolysis. This article explores the risk factors associated with development of hyponatremia and rhabdomyolysis.

Methods.-Data on the 5 cases were collected retrospectively from the runners and medical records, and all race participants were invited to complete a post-race survey. Characteristics of the cases were compared with the other race participants.

Results.-The 5 runners developing hyponatremia with rhabdomyloysis were men with a mean $( \pm$ SD) age of $39 \pm 7$ years. Presenting complaints included neurological symptoms among 3 and nausea among 3. Compared with those runners not developing hyponatremia with rhabdomyolysis, the cases tended to be younger, faster, more likely to have sustained an injury which interfered with training prior to the race, and were more likely to use nonsteroidal anti-inflammatory drugs (NSAIDs) during the race. The 3 of 5 cases progressing to acute renal failure were characterized by higher initial blood urea nitrogen (BUN; $43-69$ vs $18-23 \mathrm{mg} / \mathrm{dL}$ ) and creatinine $(2.8-4.9 \mathrm{vs} 1.1-1.2 \mathrm{mg} / \mathrm{dL}$ ) levels but were not distinguished by creatinine phosphokinase (CPK) concentrations.

Conclusions.-Over $1 \%$ of participants in the 2009 WSER were hospitalized with hyponatremia in combination with rhabdomyolysis. These individuals tended to be younger, faster, more likely to have had an injury that interfered with training, and more likely to have used NSAIDs during the race. Higher initial BUN and creatinine levels, but not CPK concentrations, distinguished those 3 who progressed to acute renal failure.
\end{abstract}

Key Words: hyponatremia, rhabdomyolysis, exercise, acute renal failure

\section{Introduction}

Only a handful of cases of exertional rhabdomyolysis in combination with exercise-associated hyponatremia (EAH) have been reported to date. Lang ${ }^{1}$ reported on a single case from the 1982 Western States Endurance Run (WSER) while Putterman et $\mathrm{al}^{2}$ reported another singular occurrence in a hiker 11 years later. More recently, Ellis

Presented at the American College of Sports Medicine annual meeting, June 2, 2010, Baltimore, MD.

Corresponding author: Martin D. Hoffman, MD, Department of Physical Medicine \& Rehabilitation (117), Sacramento VA Medical Center, 10535 Hospital Way, Mather, CA 95655-1200 (e-mail: martin. hoffman@va.gov). et $\mathrm{al}^{3}$ reported on four cases following a single $153-\mathrm{km}$ mountain footrace, while Glace and Murphy reported on a case of critical hyponatremia in a half-marathon runner who subsequently developed rhabdomyolysis secondary to combative behavior and seizure activity secondary to hyponatremic encephalopathy. ${ }^{4}$ Four of these 7 reported cases (57\%) suffered renal impairment secondary to rhabdomyolysis, while $6(86 \%)$ of these total cases presented with mental status changes secondary to hyponatremia. Thus, this relatively high percentage of medical complications underscores the potential for increased morbidity when these 2 exercise-associated conditions coexist. 
The simultaneous presentation of EAH and rhabdomyolysis creates a complicated and opposing treatment paradox with regard to fluid resuscitation. The appropriate management of EAH is either water restriction (in mild cases without neurological symptoms) or administration of a concentrated intravenous bolus of hypertonic saline (in severe cases with encephalopathy) to offset life-threatening cerebral edema. ${ }^{5}$ Conversely, the appropriate treatment for rhabdomyolysis is aggressive intravenous isotonic fluid administration to prevent myoglobin buildup in the kidneys and prevent acute renal failure (ARF). ${ }^{5}$ Therefore, field and hospital clinicians must delicately balance aggressive fluid resuscitation with mindful fluid restriction when treating symptomatic athletes presenting with such complex pathophysiologies as EAH with rhabdomyolysis.

Whether or not the combination of EAH plus rhabdomyolysis is causal or an independent association is currently under debate. According to one causal perspective, EAH may facilitate rhabdomyolysis through changes in intracellular potassium and/or calcium concentrations or through hypotonic cell swelling, both of which destabilize the muscle cell membrane and facilitate lysis. ${ }^{3}$ Conversely, pronounced lysis of muscle cells from unaccustomed physical exertion ${ }^{6,7}$ and/or significant thermal strain $^{8,9}$ could lead to third spacing of fluids, which in turn can stimulate arginine vasopressin (AVP) secretion and facilitate $\mathrm{EAH}^{3}$

As mentioned above, whether or not a more causal relationship exists between EAH and rhabdomyolysis cannot be delineated from these few case reports. However, in the present analysis, we aim to expose possible biochemical, demographic, training, and symptomatic risk factors in runners who developed simultaneous EAH and rhabdomyolysis during an ultramarathon footrace to better explore this association. We seek to uncover such "trends" by examining our small cluster of cases alone and against the larger cohort of 2009 WSER runners who did not develop the combination of EAH and rhabdomyolysis.

\section{Methods}

This case series derives from the 2009 WSER, a $161-\mathrm{km}$ ultramarathon run. The WSER course is run almost entirely on single-track mountain trials with $5500 \mathrm{~m}$ of cumulative climb and $7000 \mathrm{~m}$ of cumulative descent. Twenty-four aid stations were stocked with various fluids and nutritional items as well as salt tablets. There were no official records kept of what each participant ingested. Participants had 30 hours to complete the race to be considered an official finisher.
Local race-day ambient temperature ranged between $15^{\circ} \mathrm{C}$ and $37^{\circ} \mathrm{C}$.

Five documented cases of hyponatremia combined with rhabdomyolysis were identified from follow-up of those who were known to have received medical care during the event, and from responses to an electronic appeal sent out to all race starters by the WSER Race Director inquiring if any runners suffered any serious medical problems or were hospitalized as a consequence of race participation. Data from all 5 identified cases were collected retrospectively from hospital records plus personal (e-mail and telephone) interviews by 1 investigator (M.D.H.) within 3 weeks of race finish. The Institutional Review Board of the Department of Veterans Affairs, Northern California Health Care System approved the reporting of cases through waiver of consent.

Initial biochemical values (blood sodium, potassium, blood urea nitrogen [BUN], creatinine, creatine phosphokinase $[\mathrm{CPK}]$ ) represent the first value obtained, either from the medical tent or in the hospital. The diagnosis of ARF in this case series was based on an increase in serum creatinine $\geq 0.5 \mathrm{mg} / \mathrm{dL}$ above a presumed normal baseline or a glomerular filtration rate below $75 \%$ of the lower limit of normal. ${ }^{10}$ Body weight changes were all self-reported from serial measurements that were required at race registration, the finish and 9 checkpoints along the course. Weight measurements during the race were used to enforce the WSER weight loss policy in place at the time, which required forced rest and rehydration for a weight loss of $5 \%$ to $7 \%$.

In addition to the retrospective data collected on the 5 cases of hyponatremia combined with rhabdomyolysis, all 2009 WSER entries were sent an email as part of a larger demographic study (approved by the same Institutional Review Board) the day after the race inviting their participation in an electronic survey. Among other questions, runners were asked to indicate whether they had used nonsteroidal anti-inflammatory drugs (NSAIDs) during the race, the number of prior 161-km running races they had completed, and whether or not an injury within the past year had interfered significantly with their training. All finishers were also offered blood CPK testing at the finish as part of the routine medical services provided. These data were used to compare the 5 cases of hyponatremia and rhabdomyolysis with the remainder of the race participants. The variables of age, finish time, and prior number of $161-\mathrm{km}$ races completed were examined with unpaired $t$ tests and Cohen's effect sizes. Fisher's exact tests were used for comparing the incidence of NSAID use and presence of previous injury in the last year. Statistical significance was set at $P<.05$. 


\section{Results}

There were 400 official starters in the 2009 Western States Endurance Run (19\% women) with 240 official finishers (20\% women). Mean ( \pm SD) finishing time was $26.2 \pm 3.4$ hours $(27.3 \pm 3.2$ for women and $26.0 \pm 3.4$ for men).

\section{DESCRIPTION OF CASES}

Cases no. 1 and 4 were seen, treated and discharged from the medical tent. Case no. 1 presented with emesis, dizziness, lightheadedness, cough, and dark urine, and case no. 4 presented with dizziness, lightheadedness, and anuria. Both were treated with intravenous normal saline but then presented to the hospital within 24 hours with thigh pain and dark urine (case no. 1) and weight gain and anuria (case no. 4). Cases no. 3 and 5 were transported by ambulance after completing $150 \mathrm{~km}$ with exhaustion, confusion, severe thigh pain, dark urine, and nausea (case no. 3) and exhaustion, thigh pain, and incontinence of dark urine (case no. 5). The remaining case (no. 2) presented to the hospital 1 day after finishing the race with continued nausea and flank pain.

The five runners who developed hyponatremia with rhabdomyolysis were all male. Four cases (no. 1, 2, 4, and 5) used NSAIDs during the race. Three cases exhibited neurological symptoms at presentation (no. 1, 3, and 4), and 3 of the cases progressed to ARF (no. 1, 2, and 4). The biochemical characteristics of the 5 cases are presented in Table 1.

All 3 runners progressing to ARF finished the race, while the 2 runners who did not progress to ARF did not finish and were transported by ambulance directly off course to the hospital. When compared with the 2 sub- jects who did not progress to ARF, the 3 runners progressing to ARF had a tendency to be younger and were more likely to have lost body weight during the race (weight changes were $-4 \%,-3 \%$, and $+3 \%$ vs $+5 \%$ and $0 \%$ ) (Table 2). Those progressing to ARF also had higher initial BUN and creatinine concentrations.

\section{COMPARISON OF CASES WITH OTHER RUNNERS}

Excluding the 5 cases of hyponatremia and rhabdomyolysis, 244 men who started the race completed the post-race survey ( $76 \%$ response rate). Of these responders 158 finished the race (65\%), which was similar to the overall finish rate among men of 59\%.

Table 3 displays the comparison between the 5 cases and other men who completed the post-race survey for age, finish time, number of prior 161-km ultramarathon finishes, NSAID use during the race, and history of injury in the previous year that interfered with training. Statistical significance was not reached for any of these variables, although the $P$ value for age was .07 . Effect sizes were .8 (large) and .4 (medium) for age and finish time, respectively. Therefore, the 5 runners with documented hyponatremia and rhabdomyloysis appeared to have a collective tendency to be younger, faster, more likely to use NSAIDs and more likely to have suffered from an injury within the past year that significantly affected their ability to train.

Aside from our cases of hyponatremia and rhabdomyolysis, 59 men had CPK measurements at the finish. Of those, 12 (20\%) had CPK concentrations >38 $218 \mathrm{U} / \mathrm{L}$, the lowest initial value of the three cases progressing to ARF.

Table 1. Initial biochemical values (and range of values during treatment) in all 5 hospitalized cases of hyponatremia with rhabdomyolysis competing in the 2009 Western States Endurance Run

\begin{tabular}{|c|c|c|c|c|c|c|c|c|}
\hline $\begin{array}{l}\text { Case } \\
\text { No. }\end{array}$ & $\begin{array}{c}\text { Blood }\left[\mathrm{Na}^{+}\right] \\
\mathrm{mmol} / \mathrm{L} \\
(\text { range })\end{array}$ & $\begin{array}{c}\text { Blood }\left[K^{+}\right] \\
\text {mmol/L } \\
\text { (range) }\end{array}$ & $\begin{array}{c}\text { Blood BUN } \\
m g / d L \\
\text { (range) }\end{array}$ & $\begin{array}{c}\text { Blood } \\
\text { Creatinine } \\
\text { mg/dL } \\
\text { (range) }\end{array}$ & $\begin{array}{c}\text { Blood CPK U/L } \\
(\text { range })\end{array}$ & Urinalysis & $\begin{array}{l}\text { Days in } \\
\text { hospital }\end{array}$ & $\begin{array}{c}\text { Developed } \\
\text { acute } \\
\text { renal } \\
\text { failure }\end{array}$ \\
\hline 1 & $134(128-135)$ & $4.9(3.5-4.9)$ & $54(54-113)$ & $3.1(3.1-10.3)$ & $\begin{array}{c}>40000 \\
(9029-785250)\end{array}$ & $\begin{array}{l}+3 \text { blood } \\
+ \text { protein }\end{array}$ & 12 & Yes \\
\hline 2 & $129(129-142)$ & $3.4(3.4-3.9)$ & 69 (33-69) & $4.9(2.0-4.9)$ & $\begin{array}{c}38218 \\
(6078-38218)\end{array}$ & $\begin{array}{l}+3 \text { blood } \\
\text { trace protein }\end{array}$ & 3 & Yes \\
\hline 3 & 127 (127-139) & $3.6(3.6-4.2)$ & $23(14-23)$ & $1.2(1.0-1.2)$ & $\begin{array}{c}95940 \\
(35912-95940)\end{array}$ & $\begin{array}{l}+4 \text { blood } \\
+1 \text { protein }\end{array}$ & $<2$ & No \\
\hline 4 & $131(131-143)$ & $4.4(4.1-4.8)$ & $43(39-46)$ & $2.8(2.7-3.2)$ & $\begin{array}{c}>40000 \\
(19534->40000)\end{array}$ & $\begin{array}{l}+1 \text { blood } \\
0 \text { protein }\end{array}$ & 1 & Yes \\
\hline 5 & $131(131-140)$ & $3.9(3.6-3.9)$ & $18(10-18)$ & $1.1(1.1-1.2)$ & $\begin{array}{c}40095 \\
(17950-40095)\end{array}$ & $\begin{array}{l}+4 \text { blood } \\
+2 \text { protein }\end{array}$ & 1 & No \\
\hline
\end{tabular}


Table 2. Demographics, training history, symptoms, weight change, and biochemical values in hospitalized cases of hyponatremia with rhabdomyolysis who did $(n=3)$ and did not $(n=2)$ progress to acute renal failure after the 2009 Western States Endurance Run

\begin{tabular}{lcc}
\hline \multicolumn{1}{c}{ Variable } & Progressing to acute renal failure & Not progressing to acute renal failure \\
\hline Age (years) & $36.7 \pm 7.8$ & $42.0 \pm 7.1$ \\
Finish time (hours) & $24.3 \pm 4.6$ & Did not finish \\
Prior 161-km ultramarathon finishes (no.) & $5.3 \pm 6.8$ & $2.5 \pm 3.5$ \\
Change in body weight (\%) & $-1.3 \pm 3.8$ & $2.5 \pm 3.5$ \\
Initial blood $\left[\mathrm{Na}^{+}\right.$] (mmol/L) & $131.3 \pm 2.5$ & $129.0 \pm 2.8$ \\
Initial blood [K ${ }^{+}$] (mmol/L) & $4.2 \pm 0.8$ & $3.8 \pm 0.2$ \\
Initial blood CPK (U/L) & $218->40000$ & $4095-95940$ \\
Initial blood BUN (mg/dL) & $43-69$ & $18-23$ \\
Initial blood creatinine (mg/dL) & $2.8-4.9$ & $1.1-1.2$ \\
Presence of nausea (\% of cohort) & 67 & 50 \\
Injury interfering with training (\% of cohort) & 100 & 50 \\
\hline
\end{tabular}

Data are presented as mean $\pm \mathrm{SD}$, range or percentage.

\section{Discussion}

Both EAH and rhabdomyolysis-induced ARF are lifethreatening conditions. Current and historic consensus suggests that EAH is largely associated with overhydration $^{5}$ while ARF is commonly associated with volume depletion. $8,11,12$ These associations would collectively suggest that this pathophysiological combination would not only be paradoxical but an extremely rare event. Thus, in contrast to the apparent paradox, this report represents the largest cluster of EAH with rhabdomyolysis documented within a single athletic event to date. Although the small sample size limits the statistical findings, a few trends appear to emerge that may assist in the identification of possible risk factors that may characterize those runners predisposed to developing this complex and unusual combination.

Compared to the overall 2009 WSER cohort of men who were not hospitalized with EAH and rhabdomyolysis, the 5 men who developed this significant medical combination were younger (by about 7 years), faster (by about 2 hours), and more likely to have suffered a sig- nificant injury which curtailed proper training (a $24 \%$ higher frequency) (Table 3). Collectively, these traits would suggest that the hospitalized runners were relatively undertrained but highly motivated to complete their predetermined goal. Such mental and physical attributes could potentially facilitate the development of significant rhabdomyolysis from an overpowering mental determination to override physical discomfort leading to exceptional skeletal muscle breakdown that is already commonly associated with extreme running events. ${ }^{13-15}$ Thus, the rhabdomyolysis could have facilitated oliguria and ARF, ${ }^{16}$ which may have secondarily led to an accompanying dilutional hyponatremia.

Conversely, the 5 cases of EAH with rhabdomyolysis requiring hospitalization appeared more likely to have ingested NSAIDs (30\% higher frequency) during the race compared with the collective cohort of male 2009 WSER runners (Table 3). NSAIDs have been reported to potentiate the action of AVP at the collecting duct of the kidney. ${ }^{17}$ Additionally, 3 of the cases reported nausea. Nausea is one of the most powerful known non-osmotic

Table 3. Comparison of cases of hyponatremia and rhabdomyolysis $(n=5)$ with other male starters (non-cases) of the 2009 Western States Endurance Run who completed the post-race survey $(\mathrm{n}=244)$

\begin{tabular}{lcc}
\hline \multicolumn{1}{c}{ Variable } & Cases mean \pm SD (range) & Non-cases mean \pm SD (range) \\
\hline Age (years) & $38.8 \pm 7.2(28-47)$ & $45.4 \pm 9.7(24-72)$ \\
Finish time (hours) & $24.3 \pm 4.6(21.2-29.5)$ & $26.0 \pm 3.4(16.9-30.6)$ \\
Prior 161-km ultramarathon finishes (number) & $4.2 \pm 5.4(0-13)$ & $4.5 \pm 6.5(0-49)$ \\
NSAID use during race (\% of cohort) & 80 & 50 \\
Injury in past year that interfered with training (\% of cohort) & 80 & 56
\end{tabular}

Finish time data represent the 3 finishers among the cases and the 158 survey responders who finished the race for the non-cases. Data are presented as mean $\pm \mathrm{SD}$ and range, or percentage. 
stimuli to AVP secretion. ${ }^{18}$ Collectively, these 2 factors could potentially facilitate the development of EAH as a variant of the syndrome of inappropriate antidiuretic hormone secretion (SIADH). Thus, it is also plausible that SIADH-induced hyponatremia facilitated muscle cell membrane instability leading to concomitant rhabdomyolysis as the primary pathophysiological mechanism, as hypothesized in the polydipsic psychiatric population. ${ }^{19}$

It may be speculated that the 3 runners who subsequently pushed themselves to finish the race (and later developed ARF) may have incurred greater skeletal muscle damage by completing the maximal distance. However, the CPK results would refute this possibility. In general, the initial CPK values did not appear higher in the finishers than the nonfinishers, with the exception of case no. 1 who had a peak CPK of $785250 \mathrm{U} / \mathrm{L}$. In addition, $20 \%$ of those undergoing CPK measurements and not requiring hospitalization had concentrations higher than the lowest value of the 3 cases developing ARF. It has been emphasized that neither the presence $^{13-15}$ or severity ${ }^{20,21}$ of CPK levels associated with exercise-induced rhabdomyolysis have predictive value in either clinical outcome or progression to $\mathrm{ARF},{ }^{22,23}$ although CPK levels have been shown to be a predictor of clinical course in nonexercising scenarios. ${ }^{24}$ Our findings are consistent with the former premise. Nonetheless, the subset of runners who went on to develop ARF compared with those who did not develop ARF displayed clear signs of renal compromise through higher initial BUN (43-69 vs $18-23 \mathrm{mg} / \mathrm{dL}$ ) and creatinine (2.8-4.9 vs $1.1-1.2 \mathrm{mg} / \mathrm{dL})$ values, irrespective of the underlying cause.

One obvious difference between those runners who did or did not develop ARF is that each of the runners progressing to ARF finished the race and left the event (rather than being transported directly to the hospital) before voluntarily presenting to the emergency room within the next 24 hours when their symptomatology had not improve or had worsened. Thus, the impact of early aggressive treatment in the hospital may have prevented the progression to ARF in the 2 nonfinishers. ${ }^{25}$ The 2 runners who did not progress to ARF also reported weight gain or stable weight during the run opposed to 2 of the 3 runners who progressed to ARF. This may also suggest that the runners who did not develop ARF were overhydrated.

Our attempt to uncover trends to elucidate risk factors towards possible pathophysiological mechanisms associated with the development of EAH with rhabdomyolysis (and progression to ARF) is important for the creation of preventative strategies for future ultramarathon runners. The absence of women developing this "rare" combina- tion in this particular case series likely reflects the overall ratio of females to males (1:5) who started and finished this race, as women have developed EAH with rhabdomyolysis in previous reports. ${ }^{3,4}$

The most appropriate treatment strategy in the field and hospital setting has not been determined. In this cluster of 5 cases we are limited not only in our inability to delineate a causal relationship between EAH and rhabdomyolysis but also by the various treatments each runner received over a varying clinical course and timeline. It is important to note that one consistent feature was that all 5 cases received intravenous normal saline as part of their initial treatment; cases no. 1 and 4 at the finish line, cases no. 3 and 5 upon transfer from the $150-\mathrm{km}$ mark to the hospital, and case no. 2 the following day upon hospital presentation. Based on our current understanding, we recommend extreme caution in the use of intravenous normal saline as the initial treatment of athletes with combined EAH and rhabdomyolysis, particularly if there are any symptoms suggestive of cerebral encephalopathy, which might be expected to worsen with the use of normal saline alone. We suspect that the present cases had satisfactory outcomes with intravenous normal saline because they did not have impending cerebral encephalopathy. Clearly, further work is required to clarify optimal management of these types of cases.

In a follow-up investigation, we aim to administer 100 -mL boluses of $3 \%$ hypertonic saline to runners with documented EAH to determine if this strategy elicits a rapid return to normonatremia and reduces the incidence of ARF in those with concomitant rhabdomyloysis subsequently treated with copious isotonic saline administration.

Limitations to this small retrospective cohort study include the fact that we were required to rely on selfreported body weights from multiple scales for which accuracy was unknown. Details on fluid and nutrition intake during the event, and the extent of heat acclimatization among the runners were also unknown.

In conclusion, 5 runners ( $1 \%$ of the starting field) participating in the 2009 161-km WESR were admitted to the hospital with EAH in combination with rhabdomyolysis. These hospitalized runners had a tendency towards being younger, faster, having sustained a previous injury which interfered with their training, and being more likely to ingest NSAIDs than those male competitors who did not develop this disorder. We suggest that clinicians be aware that EAH and rhabdomyolysis may coexist in athletes participating in ultramarathon footraces and be mindful that the treatment for each entity is different and theoretically contradictory. 


\section{Acknowledgments}

The authors thank Greg Soderlund, the Race Director of the Western States Endurance Run, for his assistance in identifying the cases reported here, and the runners who have shared of themselves to advance the safety of the sport.

\section{References}

1. Lang G. Medical findings in the Western States 100 Mile. Ultrarunning. 1984;3:14-18.

2. Putterman C, Levy L, Rubinger D. Transient exerciseinduced water intoxication and rhabdomyolysis. Am J Kidney Dis. 1993;21:206-209.

3. Ellis C, Cuthill J, Hew-Butler T, George SM, Rosner MH. Case report: exercise-associated hyponatremia with rhabdomyolysis during endurance exercise. Phys Sportsmed. 2009;37:126-132.

4. Glace B, Murphy C. Severe hyponatremia develops in a runner following a half-marathon. J Am Acad Physician Assist (JAPPA). 2008;21:27-29.

5. Hew-Butler T, Ayus JC, Kipps C, et al. Statement of the second international exercise-associated hyponatremia consensus development conference, New Zealand, 2007. Clin J Sport Med. 2008;18:111-121.

6. Clarkson PM, Kearns AK, Rouzier P, Rubin R, Thompson PD. Serum creatine kinase levels and renal function measures in exertional muscle damage. Med Sci Sports Exerc. 2006;38:623-627.

7. Gupta A, Khaira A, Tiwari SC. Hyponatremia, rhabdomyolysis and acute kidney injury. Saudi J Kidney Dis Transplant. 2009;20:674.

8. Vertel RM, Knochel JP. Acute renal failure due to heat injury. An analysis of ten cases associated with a high incidence of myoglobinuria. Am J Med. 1967;43:435-451.

9. de Souza AL, Rodrigues C, Sztajnbok J, et al. Hypoelectrolytemia accompanied by acute renal failure in exertional heatstroke. Am J Emerg Med. 2006;24:888-889.

10. Bellomo R, Ronco C, Kellum JA, Mehta RL, Palevsky P. Acute renal failure-definition, outcome measures, animal models, fluid therapy and information technology needs: the Second International Consensus Conference of the Acute Dialysis Quality Initiative (ADQI) Group. Crit Care. 2004;8:R204-R212.
11. Irving RA, Noakes TD, Raine RI, Van Zyl Smit R. Transient oliguria with renal tubular dysfunction after a $90 \mathrm{~km}$ running race. Med Sci Sports Exerc. 1990;22:756-761.

12. Bosch X, Poch E, Grau JM. Rhabdomyolysis and acute kidney injury. $N$ Engl J Med. 2009;361:62-72.

13. Dumke CL, Nieman DC, Oley K, Lind RH. Ibuprofen does not affect serum electrolyte concentrations after an ultradistance run. Br J Sports Med. 2007;41:492-496.

14. Schiff HB, MacSearraigh ET, Kallmeyer JC. Myoglobinuria, rhabdomyolysis and marathon running. $Q \mathrm{~J} \mathrm{Med}$ 1978;47:463-472.

15. Skenderi KP, Kavouras SA, Anastasiou CA, Yiannakouris $\mathrm{N}$, Matalas AL. Exertional rhabdomyolysis during a 246-km continuous running race. Med Sci Sports Exerc. 2006;38:1054-1057.

16. MacSearraigh E, Kallmeyer J, Schiff H. Acute renal failure in marathon runners. Nephron. 1979;24:236-240.

17. Walker RJ, Fawcett JP, Flannery EM, Gerrard DF. Indomethacin potentiates exercise-induced reduction in renal hemodynamics in athletes. Med Sci Sports Exerc. 1994;26: 1302-1306.

18. Rowe JW, Shelton RL, Helderman JH, Vestal RE, Robertson GL. Influence of the emetic reflex on vasopressin release in man. Kidney Int. 1979;16:729-735.

19. Cronin RE. Psychogenic polydipsia with hyponatremia: report of eleven cases. Am J Kidney Dis. 1987;9:410-416.

20. Lonka L, Pedersen RS. Fatal rhabdomyolysis in marathon runner. Lancet. 1987;1:857-858.

21. Hurley JK. Severe rhabdomyolysis in well conditioned athletes. Mil Med. 1989;154:244-245.

22. Sinert R, Kohl L, Rainone T, Scalea T. Exercise-induced rhabdomyolysis. Ann Emerg Med. 1994;23:1301-1306.

23. Clarkson PM. Exertional rhabdomyolysis and acute renal failure in marathon runners. Sports Med. 2007;37: 361-363.

24. de Meijer AR, Fikkers BG, de Keijzer MH, van Engelen BG, Drenth JP. Serum creatine kinase as predictor of clinical course in rhabdomyolysis: a 5-year intensive care survey. Intensive Care Med. 2003;29:1121-1125.

25. Ron D, Taitelman U, Michaelson M, Bar-Joseph G, Bursztein S, Better OS. Prevention of acute renal failure in traumatic rhabdomyolysis. Arch Intern Med. 1984;144: 277-280. 